\title{
Probioticos y la enfermedad periodontal: Revisión de la Literatura
}

\section{Probiotics and periodontal disease: Review of the Literature}

\section{Britto Ebert Falcón Guerrero ${ }^{1}$}

1. Doctorado en Estomatología, Magister en odontoestomatología, Especialista en Periodoncia e Implantología, Practica Privada.

\section{RESUMEN}

Los probióticos representan una alternativa para mantener la salud mediante la utilización de las bacterias naturales beneficiosas. Estos han sido muy estudiados por lograr mejorar la salud del sistema digestivol. Sin embargo, en los últimos años se vienen haciendo investigaciones en el mantenimiento y el tratamiento de la enfermedad periodontal. En este trabajo se resume los datos actualmente disponibles sobre los beneficios potenciales de los probióticos en el periodonto.

Palabras claves: Probióticos, enfermedad periodontal, prevención, tratamiento.

\begin{abstract}
Probiotics represent an alternative to maintain health using beneficial natural bacteria. These have been well studied for improving the health of the digestive system. However, in recent years research has done on the maintenance and treatment of periodontal disease. This paper summarizes the currently available data on the potential benefits of probiotics in the periodontium.
\end{abstract}

Key words: Probiotics, periodontal disease, prevention, treatment.

enfermedad. Por lo que, esta hipótesis plantea nuevas estrategias dirigidas a potenciar un ambiente saludable y mantener el equilibrio simbiótico de la bio-película ${ }^{4}$.

En el periodonto, debemos tener en cuenta la etiología de la enfermedad periodontal relacionada con la placa bacteriana. Socranszky, Considera tres factores que determinan el desarrollo de la enfermedad: Huésped susceptible, presencia de patógenos y la reducción o ausencia de bacterias benéficas ${ }^{5,6,7}$.

En un tratamiento tradicional, se debe tener en cuenta que el cambio hacia una microbiota menos patógena, es sólo temporal, restableciéndose una microbiota más agresiva dentro de semanas o meses $^{8}$. Esta re-colonización depende del nivel de higiene bucal, la eficacia del raspado y alisado radicular (RAR) y las profundidades al sondaje de las bolsas residuales ${ }^{2,6 .}$ 
Esta terapia va acompañada de antibioticoterapia sistémica, que elimina toda la microbiota, independiente de su patogenicidad; aumentando la resistencia bacteriana con cepas multiresistentes y la recolonización de los sitios tratados?.

La distribución específica de bacterias que colonizan la bolsa periodontal puede ser alterada con terapia de reemplazo, conocida como terapia probiótica, protegiéndo a través de la promoción de la respuesta beneficiosa del huésped ${ }^{7}$.

Teughels y $\mathrm{col}^{1}$ proponen el concepto de una recolonización guiada de la bolsa periodontal para tratar la infección periodontal; su estudio reveló que cuando se aplicaron bacterias benéficas en las bolsas periodontales después del RAR, la recolonización subgingival con periodontopatógenos disminuyo significativamente, así como el proceso de inflamación. Tomando en cuenta estos antecedentes, se realiza una revisión de la literatura actualizada sobre los probióticos relacionados con la enfermedad periodontal, que es una de las enfermedades de mayor prevalencia en la boca de los seres humanos.

\section{PROBIÓTICOS}

Es una nueva palabra para nombrar bacterias con efecto beneficioso para humanos, que proviene del griego que se traduce como "ayuda o que favorece a la vida". Su antónimo vendría a ser el término antibiótico ${ }^{10}$.

Elie Metchnikoff, vio que los campesinos búlgaros eran más longevos y pensó que era por ingerir derivados lácteos fermentados por bacterias. Proponiendo que estas bacterias, de estos productos lácteos, competían con bacterias dañinas para la salud ${ }^{11}$. Metchnikoff descubrió el Lactobacilo bulgaricus, cepa que es utilizada para producir productos ácido-lácteos ${ }^{1}$.

El termino Probiótico, es introducido por Lilly y stillwell $(1965){ }^{10}$ y lo definen como una sustancia producida por microorganismos para promover el crecimiento de otros microorganismos.

En 1991, Holocombh fue el primero en investigar al Bifidobacterium bifidum como especie probiótica ${ }^{11}$.

La OMS a través de la FAO (2001) ${ }^{12}$, los define de la siguiente manera: microorganismos vivos que cuando se administra en proporciones óptimas, proporcionan un beneficio para la salud del hospedero.
Guarner y col. (2005), consenso europeo de Life Science Institute, propuso una definición simple y ampliamente aceptada: suplementos alimentarios microbianos viables que influyen positivamente en la salud de los seres humanos. ${ }^{2,13}$

PREBIÓTICO: El término fue introducido por Gibson y Roberfroid que intercambiaron "pro" por "pre" que significa "antes" o "por" ${ }^{1}$. Son ingredientes alimenticios no digeribles que brindan efectos benéficos en el hospedero, produciendo un crecimiento y actividad selectiva de una especie limitada de microorganismos del colon. Esta definición es también sinónima de la fibra dietética. Destacando los fructo-oligosacaridos y los galacto-oligosacaridos ${ }^{2,14}$. Dentro de ellos los más prometedores son el betametil-D-galactosido y la $\mathrm{N}$-acetil-D-manosamina, dependiendo cada uno de ellos de vías metabólicas específicas y selectivas ${ }^{15}$.

Slomka y $\operatorname{col}^{15}$, concluyen que los prebióticos producen una estimulación nutricional de las bacterias beneficiosas, que podría ser utilizada para restaurar el equilibrio microbiano y por esto promover la salud oral.

SIMBIÓTICOS: Son productos compuestos por probióticos y prebióticos. Donde el prebiótico favorece selectivamente a un probiótico especifico. Dándose un efecto de sinergismo directo entre ambos componentes ${ }^{2,16}$.

Dentro de los probióticos más usados y estudiados están las especies de Lactobacilos y Bifidobacterium ${ }^{11}$, y su característica general es su origen humano ${ }^{6,14}$. Sin embargo, se debe tener en cuenta que todos los probióticos no tienen la misma eficacia ${ }^{14}$. Por ejemplo: el Lactobacilos rhamnosus GG, reduce el riesgo de caries, produciendo una sustancia inhibidora del crecimiento contra Streptococcus sobrinus. ${ }^{2}$, así también, el Lactobacilos reuteri reduce el número de estreptococos mutans ${ }^{11}$.

Las bacterias probióticas promueven la salud del huésped mediante la exclusión competitiva de las bacterias patógenas, brindan nutrientes y cofactores, compiten con los patógenos inhibiendo su crecimiento, interactúan con los factores de virulencia, brindan permeabilidad epitelial y estimulan la respuesta inmune del hospedero ${ }^{14,17}$. Por ejemplo, las cepas de Lactobacilos rhamnosus y lactobacilos paracasei poseen fuerte capacidad de adhesión a las superficies bucales (más que el $20 \%$ que otras bacterias), lo cual les 
permite ser los primeros colonizadores proveyendo un biofilm saludable ${ }^{11}$

\section{FORMA DE ACCIÓN DE LOS PROBIÓTICOS}

Estas acciones o efectos de los probióticos se dan a partir de tres modos de acción ${ }^{1,10,13}$ : (1) la modulación de las defensas del huésped incluyendo el sistema inmune innato y adquirido: los probióticos pueden potenciar la expresión de los receptores de la fagocitosis en los neutrófilos de individuos sanos y aumentan la actividad de los natural killers. Modulando así, tanto la respuesta inmune innata como la adaptativa. (2) Producción de sustancias antimicrobianas, como: ácido láctico, peróxido de hidrógeno, bacteriocinas. Los ácidos lácticos pueden pasar a través de las membranas celulares bacterianas y acidificar el citoplasma, y a su vez puede inhibir la proliferación bacteriana. Teniendo actividad antimicrobiana contra Porfiromonas gingivalis $y$ Streptococos mutans. El Peróxido de hidrógeno inhibe el crecimiento de especies bacterianas patógenas, como Agregatibacter actinomicentemcomitans; y las Bacteriocinas, son péptidos catiónicos sintetizados ribosomalmente, que actúa frente a Prevotella spp, responsables de la halitosis y puede ser capaz de matar a la Porfiromonas gingivalis. (3) Mecanismos de exclusión competitiva. El principio de exclusión competitiva, también conocido como la ley de Gause, establece que dos especies que compiten por los mismos recursos no pueden coexistir de manera estable. Uno de los dos competidores siempre tendrá una ligera ventaja sobre el otro que lleva a la extinción del segundo competidor o un cambio de esta especie a otro nicho. El mecanismo de exclusión competitiva utilizado por las bacterias beneficiosas puede ocurrir en dos niveles: Dificultando la adhesión de bacterias patógenas o Compitiendo por los mismos nutrientes.

\section{EFECTOS ADVERSOS Y SEGURIDAD}

La seguridad de los probióticos es preocupante, debido al exceso de suplementos en diferentes productos alimenticios. Como medida de seguridad, las bacterias probióticas deben ser: no patógenas, no deben causar efecto estimulante del crecimiento de bacterias patógenas, no deben transferir genes resistentes a los antibióticos y los probióticos deben mantener el ecosistema de la microflora ${ }^{11}$.

Los lactobacilos y los bifidobacteriums se encuentran en la alimentación y en un intestino sano. Por lo que, podrían ocasionar enfermedades espontáneas, sin tener que contaminarse necesariamente con estos. Los probióticos se consideran seguros y bien tolerados; pero cuando se ingieren por vía oral puede aparecer con frecuencia, hinchazón y flatulencia ${ }^{1}$,

Se han reportado pocos casos como: bacteriemia, sepsis o endocarditis por lactobacilos rhamnosus GG o Lactobacilos casei. Todos los casos de infecciones por probióticos se han presentado cuando el paciente tiene alguna enfermedad condicionante, como: Diabetes mellitus, valvulopatías, recién nacidos prematuros, alteraciones sanguíneas, síndrome de inmunodeficiencia adquirida, pacientes en cuidados intensivos y con alimentación parenteral, pacientes trasplantados y con cáncer, etc. Sin embargo, casi todos los casos de bacteriemia probiótica responden bien a una terapia antibiótica apropiada ${ }^{1}$.

\section{TRATAMIENTO DE LA ENFERMEDAD PERIODONTAL}

Las enfermedades periodontales se clasifican en dos tipos: gingivitis y periodontitis. La gingivitis es la inflamación de la encía, mientras que la periodontitis es una enfermedad progresiva y destructiva que daña al sistema de soporte periodontal, incluyendo el hueso alveolar ${ }^{11}$.

\section{GINGIVITIS Y FORMACIÓN DE PLACA}

Las especies de lactobacilos más frecuentes detectadas en sujetos sanos fueron Lactobacilos gasseri y Lactobacilos fermentum. La presencia de Lactobacilos gasseri se asoció con menos placa dental y menos inflamación gingival. Interfieren con el metabolismo y/o crecimiento de los patógenos putativos periodontales, afectando así las interacciones huésped-microbio ${ }^{14}$.

Karuppaiah y col. ${ }^{18}$, administraron por 30 días a niños con gingivitis una bacteria de ácido láctico, encontrando una reducción estadísticamente significativa en la formación de la placa bacteriana; mejorando la salud gingival.

Iniesta y $\mathrm{col}^{19}$, administraron una tableta diaria entre 4 a 8 semanas, que contenían 2 cepas de Lactobacilos reuteri (DSM-17938 y ATCC PTA 5289), encontrando una reducción de la Prevotella intermedia en la saliva y una reducción de Porfiromonas gingivales en la microbiota subgingival.

Alkaya y $\mathrm{col}^{17}$, han descrito el efecto de bacilos probiòticos en la gingivitis generalizada, estos se adicionan en productos para la higiene (pasta dental, enjuague y cepillo de dientes) conteniendo: Bacilos 
subtilis, Bacilos megaterium y Bacilos pumulus; estos mostraron ciertas mejoras, pero no encontraron diferencias significativas en comparación con productos tradicionales.

Amižić y $\mathrm{col}^{20}$, realizaron pruebas con pastas dentales y enjuagues, una contenía Lactobacilos paracasei y el otro contenía Lactobacilos acidophilus. Las cremas dentales probióticas tuvieron mejor efecto inhibitorio en el caso de Candida albicans y Streptococos salivarius. En todos los casos, las pastas dentales tenían una capacidad de inhibición más fuerte que los enjuagues bucales. Estos resultados indican que pueden usarse en la prevención de enfermedades infecciosas orales.

Con respecto a la respuesta del huésped en la modulación del proceso inflamatorio, Lee y col ${ }^{21}$, estudiaron al Lactobacilos brevis CD2, quien puede inhibir la inflamación gingival de origen natural, mediante la prevención de la síntesis de óxido nítrico reduciendo su presencia en el líquido crevicular y disminuyendo la formación de prostaglandinas E2, retrasando así el desarrollo de la gingivitis.

\section{PERIODONTITIS}

Los principales agentes patógenos asociados con la periodontitis son Porfiromonas gingivalis, Treponema denticola, Aggregatibacter actinomycetemcomitans y Tannerella forsythia. Estas bacterias tienen un carácter virulento que varía en la colonización de los sitios subgingivales, escapan del sistema de defensa hasta producir daño tisular. La persistencia de la respuesta inmune del huésped es también un factor determinante en la progresión de la enfermedad ${ }^{11}$.

Messora y col $^{22}$, evaluaron los efectos de las especies de Bacilos (Subtilis y Licheniformis) en periodontitis experimental en ratas, demostrando que el uso de bacilos reduce la perdida ósea y de adherencia, proporcionando un efecto protector y mejorando los efectos del RAR en el manejo de la enfermedad periodontal.

Van Essche y col. ${ }^{23}$, reportaron que el Bdellovibrio son bacterias depredadoras (se alimentan de bacterias Gram-negativas), que se encuentran en la naturaleza, aislándolas de distintos biofilms terrestres y acuáticos, incluyendo las heces de los animales. Mencionando que la cepa Bdellovibrio bacteriovirus HD100, es capaz de atacar, alimentarse y matar al Agregatibacter actinomycetemcomitans, actuando como un antibiótico viviente, siendo útil para tratar y prevenir la periodontitis.

También, se ha reportado que los Lactobacilos disminuyen los valores de lactoferrina en el fluido gingival, obteniéndose valores similares a un estado saludable, esto es importante ya que la lactoferrina se correlaciona con los parámetros clínicos de la periodontitis; del mismo modo, se ha informado que el ácido lipoteicoico del Lactobacilo plantarum suprimió la producción de citoquinas proinflamatorias y de óxido nítrico ${ }^{24}$.

Jaffar y col. ${ }^{24}$, encontraron que bacterias como Lactobacilos spp, son capaces de degradar en un $90 \%$ Ia biopelícula del Agregatibacter actinomicentencomitans, en cepas de Agregatibacter actinomycetemcomitans Y4 (serotipo b) y Agregatibacter actinomycetemcomitans OMZ 534 (serotipo e), pero en el biofilm de la cepa del Agregatibacter actinomycetemcomitans SUNY 75 (serotipo a), no se obtuvo un gran impacto. Esta degradación del biofilm maduro se logra debido a la producción de tres enzimas: la proteasa, la lipasa y la amilasa.

Kobayashi y $\operatorname{col}^{25}$, evaluaron el tratamiento del Lactobacilos gasseri SBT2055, en ratones, para la infección por Porfiromonas gingivalis; reduciendo significativamente la pérdida ósea alveolar, perdida de adhesión, la desorganización del ligamento periodontal y la colonización bacteriana. Además, hubo una reducción significativa de la expresión y secreción del factor de necrosis tumoral- $\alpha$ y de interleucina- 6 en el tejido gingival. Potenciando la inmunorregulación innata y mejorando la prevención de la periodontitis.

Laleman y col. ${ }^{5}$, utilizaron cepas de Streptococos oralis KJ3, Streptococos uberis KJ2 y Streptococos rattus JH145, junto con el RAR para el control de la periodontitis; demostrando que a las 12 semanas hay una disminución de la profundidad al sondaje y se halla un recuento bajo de Prevotella intermedia. Dentro de ellos el Streptococos uberis produce peróxido de hidrogeno que inhibe el crecimiento del Agregatibacter actinomycetemcomitans.

Iwasaki y col. ${ }^{26}$, concluyen que la cepa de Lactobacilos plantarum L-137 ingerida en capsulas durante 12 semanas, como terapia periodontal de mantenimiento, disminuye la profundidad de las bolsas periodontales. 
Sin embargo, Yanine y $\mathrm{col}^{27}$ en el 2013 , reportan en su revisión que la efectividad de los probióticos para prevenir y tratar la enfermedad periodontal es cuestionable, debido a que no encontraron suficientes pruebas que demuestren los beneficios de uso preventivo de probióticos en pacientes con enfermedades periodontales.

\section{PERIODONTITIS CRÓNICA}

La periodontitis crónica es vista como un problema epidemiológico debido a su alta prevalencia de destrucción periodontal en la población mundial.

Matsubara y $\mathrm{col}^{28}$, en un trabajo de revisión, encontraron que los probióticos son efectivos en la periodontitis crónica, reportando una investigación que muestra una disminución tanto para: la profundidad y sangrado al sondaje, el índice gingival e índice de placa; en los grupos de probióticos $(p<0,05)$ durante un período de seguimiento a largo plazo de 360 días. Por lo que concluyen que su uso adyuvante puede mejorar los índices de la enfermedad y reducir la necesidad de antibióticos.

El consumo de Lactobacilos rhamnosus SP1 (aislado de la mucosa del intestino) en un polvo de disolución oral (inhiben el crecimiento de periodontopatógenos a través de bacteriocinas, tiene efecto inmunomodulador y por qué produce un medio acido en la placa supragingival), adicional a la terapia periodontal no quirúrgica ${ }^{9}$.

El tratamiento gold standard para la periodontitis es el RAR, cuyo objetivo es controlar la infección, erradicando los patógenos que se encuentran a nivel subgingival, para establecer una comunidad microbiana compatible con la salud del hospedero ${ }^{1,9}$. La terapia con probióticos evita la respuesta destructiva del hospedero, por lo que su uso puede ser útil para lograr el objetivo del tratamiento ${ }^{7}$.

Tekce y col. ${ }^{6}$, mencionan que el Lactobacilos reuteri es un agente adyuvante útil para retardar la recolonización y mejorar los resultados clínicos de la periodontitis crónica. El Lactobacilos reuteri, se encuentra en la saliva y placa subgingival, este forma Reuterina, que evita el crecimiento excesivo de otros microorganismos patógenos. También reprime mediadores inflamatorios tales como factor de necrosis tumoral $\alpha$, interleucina-8 e interleucina- $1 \beta$.

\section{MUCOSITIS PERI-IMPLANTARIA}

Una característica principal de la mucositis es que es un proceso inflamatorio reversible y no compromete la perdida de hueso alveolar, esta tiene una prevalencia de $43 \%$ en los implantes dentales. Por lo que es importante controlarla para que no se establezca la periimplantitis $^{29,30}$.

Se ha reportado que la ingesta de Lactobacilos plantarum y Lactobacilos brevis, han presentado buenos resultados en mucositis. Disminuyendo el sangrado al sondaje y de las citocinas pro y anti inflamatorias ${ }^{29}$.

Hallström y col. ${ }^{30}$, no encontraron que la ingesta de Lactobacilos reuteri, mejorara los resultados de la mucositis, en comparación con la terapia mecánica. Sin embargo, Flichy-Fernandez y col. ${ }^{31}$, encontraron que la ingesta del Lactobacilos reuteri prodentis durante 30 días, en pacientes con mucositis periimplantaria mostraron mejorías en los parámetros clínicos de inflamación, con reducción en los niveles de citoquinas. Siendo una buena alternativa para el tratamiento y prevención de la mucositis.

Sin embargo, recientemente Mogardini y col. ${ }^{29}$, Evaluaron la eficacia clínica de los probióticos (Lactobacilos plantarum y Lactobacilos brevis) en el tratamiento de la mucositis, con la eliminación profesional de la placa y con terapia fotodinámica. El efecto de la terapia fotodinámica, se debe principalmente a las propiedades bactericidas mostradas cuando se producen especímenes libres de oxígeno reactivo (pasando a un estado triplemente energizado) por la activación de luz en una sustancia fotosensibilizadora dentro de la bolsa, dando resultados microbiológicos y clínicos positivos para el tratamiento de la periodontitis. Ellos concluyen en que el uso de probióticos no da un resultado significativamente diferente, en la mejora del tratamiento.

\section{TENDENCIAS FUTURAS}

Las bacterias probióticas como Escherichia coli, colonizan al recién nacido; estimulaando el sistema inmune de la mucosa para producir anticuerpos e inmunoglobulinas. Los hallazgos actuales sobre el uso potencial de los probióticos contra las condiciones orales, son muy alentadores pensando en establecerlos para prevenir y tratar las infecciones orales $^{11}$. 
Mitsuoka ${ }^{32}$, introduce el concepto de los Biogénicos, que se definen como: "ingredientes alimenticios que afectan de manera beneficiosa al huésped mediante inmunoestimulación directa o supresión de mutagénesis, tumorigénesis, peroxidación, hipercolesterolemia o putrefacción intestinal"

Ohshima y col. ${ }^{16}$, mencionan que los Biogènicos, son una estrategia para superar la desventaja potencial de los simbióticos, incluyendo las dificultades en el proceso de colonización de bacterias probióticas no nativas, y también proporciona una ventaja adicional para producir alimentos funcionales con metabolitos bioactivos.
Se concluye que los probióticos representan una gran oportunidad para tratar las enfermedades periodontales de una manera natural y no invasiva. Sus propiedades han hecho que sea un tema de interés debido a que estos existen en la microflora oral normal, por lo que estas bacterias ofrecen la ventaja de estar perfectamente adaptadas. De todos modos, se necesitan más estudios para comprender la capacidad de las bacterias probióticas para sobrevivir, crecer y tener un buen efecto terapéutico; así mismo, determinar una administración y dosis adecuada como coadyuvante en el tratamiento de las enfermedades periodontales.

\section{REFERENCIAS BIBLIOGRÁFICAS}

1. Teughels W, Loozen G, Quirynen $M$ : Do probiotics offer opportunities to manipulate the periodontal oral microbiota? j clin periodontol 2011; 38 (suppl. 11): 159-177. doi: 10.1111/j.1600-051x.2010.01665.x.

2. Tandon V, Arora V, Yadav V, Singh V, Punia H, Agrawal S, Gupta V. Concept of Probiotics in Dentistry. Int J Dent Med Res 2015;1(6):206-209.

3. Marsh PD (1994) Microbial ecology of dental plaque and its significance in health and disease. Adv Dent Res 8, 263-271.

4. Zalba Elizari JI, Flichy-Fernández AJ. Empleo de probióticos en odontología. Nutr Hosp Vol. 28. Suplemento 1. 2013. 49-50.

5. Laleman I, Yilmaz E, Ozcelik O, Haytac C, Pauwels M, Herrero ER, Slomka V, Quirynen M, Alkaya B, Teughels W. The effect of a streptococci containing probiotic in periodontal therapy: a randomized controlled trial. J Clin Periodontol 2015; 42: 1032-1041. doi: 10.1111/jcpe.12464.

6. Tekce M, Ince G, Gursoy H, Dirikan Ipci S, Cakar G, Kadir T, Yilmaz S. Clinical and microbiological effects of probiotic lozenges in the treatment of chronic periodontitis: a 1-year follow-up study. J Clin Periodontol 2015; 42: 363-372. doi: 10.1111/jcpe.12387

7. Gupta ND, Sharma S, Sharma VK. Probiotic - An emerging therapy in recolonizing periodontal pocket, J Oral Biol Craniofac Res. (2016), http://dx.doi.org/10.1016/j.jobcr.2016.09.002

8. Quirynen M, Vogels R, Pauwels M. Initial subgingival colonization of 'pristine' pockets. J Dent Res. 2005;84(4):340-344.

9. Morales A, Galaz C, González J, Silva N, Hernández M, Godoy C, García-Sesnich J, Díaz P, Carvajal P. Efecto clínico del uso de probiótico en el tratamiento de la periodontitis crónica: ensayo clínico. Revista Clínica de Periodoncia, Implantología y Rehabilitación Oral, vol. 9, núm. 2, agosto, 2016, pp. 146-152

10. Teughels W, Van Essche M, Sliepen I, Quirynen M. Probiotics and oral healthcare. periodontology 2000, vol. 48, 2008, 111-147.

11. Alok $A$, Singh ID, Singh $S$, Kishore $M$, Jha $P C$, Iqubal MA. Probiotics: A New Era of Biotherapy. Adv Biomed Res 2017;6:31.

12. Schlundt J. Health and Nutritional Properties of Probiotics in Food including Powder Milk with Live Lactic Acid Bacteria. Report of a Joint FAO/WHO Expert Consultation on Evaluation of Health and Nutritional Properties of Probiotics in Food Including Powder Milk with Live Lactic Acid Bacteria 2001 [cited 201322 February]; Available from:

http://www.who.int/foodsafety/publications/fs_management/ en/probiotics.pdf

13. Mendi A, Köse S, Yılmaz D, Uçkan D. Oral probiotics for dentistry. OA Dentistry 2014 Jun 14;2(1):9.

14. Stamatova I, Meurman JH. Probiotics and periodontal Disease.Periodontology 2000, Vol. 51, 2009, 141-151
15. Slomka V, Hernandez-Sanabria E, Herrero ER, Zaidel L, Bernaerts K, Boon N, Quirynen M, Teughels W. Nutritional stimulation of commensal oral bacteria suppresses pathogens: the prebiotic concept. J Clin Periodontol. 2017 Apr;44(4):344-352. doi: 10.1111/jcpe.12700. Epub 2017 Feb 18.

16. Ohshima T, Kojima Y, Seneviratne CJ, Maeda N. Therapeutic Application of Synbiotics, a Fusion of Probiotics and Prebiotics, and Biogenics as a New Concept for Oral Candida Infections: A Mini Review. Front Microbiol. 2016 Jan 25;7:10. doi: 10.3389/fmicb.2016.00010. eCollection 2016.

17. Alkaya B, Laleman I, Keceli S, Ozcelik O, Cenk Haytac M, Teughels W. Clinical effects of probiotics containing Bacillus species on gingivitis: a pilot randomized controlled trial. J Periodont Res 2016; doi:10.1111/jre.12415.

18. Karuppaiah RM, Shankar S, Raj SK, Ramesh K, Prakash R, Kruthika $M$. Evaluation of the efficacy of probiotics in plaque reduction and gingival health maintenance among school children - A Randomized Control Trial. J Int Oral Health 2013; 5(5):33-7.

19. Iniesta $M$, Herrera $D$, Montero $E$, Zurbriggen $M$, Matos $A R$, Marıan MJ, Sanchez- Beltra'n MC, Llama-Palacio A, Sanz M. Probiotic effects of orally administered Lactobacillus reutericontaining tablets on the subgingival and salivary microbiota in patients with gingivitis. A randomized clinical trial. J Clin Periodontol 2012; doi: 10.1111/j.1600-051X.2012.01914.x.

20. Amižić IP, Cigić2 L, Gavić2 L, Radić M, Lukenda2 DB, Tonkić M, Barišić IG. Antimicrobial Efficacy of Probiotic-Containing Toothpastes: An in Vitro Evaluation. Med Glas (Zenica) 14 (1), 139-144.2017 Feb 01.

21. Lee JK, Kim SJ, Ko SH, Ouwehand AC, Ma DS. Modulation of the host response by probiotic Lactobacillus brevis CD2 in experimental gingivitis. Oral Dis. 2015 Sep;21(6):705-12. doi: 10.1111/odi.12332. Epub 2015 Apr 20.

22. Messora MR, Pereira LJ, Foureaux R, Oliveira LFF, Sordi CG, Alves AJN, Napimoga MH, Nagata MJH, Ervolino E, Furlaneto FAC. Favourable effects of Bacillus subtilis and Bacillus licheniformis on experimental periodontitis in rats. Arch Oral Biol 2016 . 66, 108-119.

23. Van Essche, Quirynen, Sliepen I, Van Eldere J, Teughels. Bdellovibrio bacteriovorus attacks Aggegatibacter actinomycetemcomitans. J Dent Res, 2009; 88(2): 182-186.

24. Jaffar N, Ishikawa $\mathrm{Y}$, Mizuno $\mathrm{Y}$, Okinaga T, Maeda T. Mature Biofilm Degradation by Potential Probiotics: Aggregatibacter actinomycetemcomitans versus Lactobacillus spp. PLoS One. 2016; 11(7): e0159466.

25. Kobayashi R, Kobayashi T, Sakai F, Hosoya T, Yamamoto M, KuritaOchiai T. Oral administration of Lactobacillus gasseri SBT2055 is effective in preventing Porphyromonas gingivalis-accelerated 
periodontal disease. Sci Rep. 2017 Apr 3;7(1): 545. doi: 10.1038/s41598-017-00623-9.

26. Iwasaki K, Maeda K, Hidaka K, Nemoto K, Hirose Y, Deguchi S. Daily Intake of Heat-killed Lactobacillus plantarum L-137 Decreases the Probing Depth in Patients Undergoing Supportive Periodontal Therapy. Oral Health Prev Dent. 2016;14(3):207-14. doi: 10.3290/j.ohpd.a36099.

27. Yanine N, Araya I, Brignardello-Petersen R, Carrasco-Labra A, González A, Preciado A, Villanueva J, Sanz M, Martin C. Effects of probiotics in periodontal diseases: A systematic review. Clin Oral Investig. 2013 Sep;17(7):1627-34. doi: 10.1007/s00784-0130990-7. Epub 2013 May 9.

28. Matsubara VH, Bandara HMHN, Ishikawa KH, Mayer MPA, Samaranayake LP. The role of probiotic bacteria in managing periodontal disease: a systematic Review. Expert Rev Anti Infect Ther. 2016 Jul;14 (7): 643-55. doi: 10.1080 /14787210.2016.1194198. Epub 2016 Jun 3.

29. Mongardini C, Pilloni A, Farina R, Di Tanna G, Zeza B. Adjunctive efficacy of probiotics in the treatment of experimental periimplant-mucositis with mechanical and photodynamic therapy: a randomized, cross-over clinical trial. J Clin Periodontol 44 (4), 410-417. 2017 Feb 18. doi: 10.1111/jcpe.12689.

30. Hallström $\mathrm{H}$, Lindgren $\mathrm{S}$, Widén $\mathrm{C}$, Renvert $\mathrm{S}$, Twetman S. Probiotic supplements and debridement of peri-implant mucositis: a randomized controlled trial. Acta Odontol Scand. 2016;74(1):60-6. doi: 10.3109/00016357.2015.1040065. Epub 2015 May 8.

31. Flichy-Fernandez AJ, Ata-Ali J, Alegre-Domingo T, Candel-Marti E, Ata-Ali F, Palacio JR, Peñarrocha-Diago M. The effect of orally administered probiotic Lactobacillus reuteri-containing tablets in peri-implant mucositis: a double-blind randomized controlled trial. J Periodont Res 2015; 50: 775-785.

32. Mitsuoka T. Significance of dietary modulation of intestinal flora and intestinal environment. Bioscience Microflora, 2000Vol. 19 (1), 15-25.

\section{Correspondencia}

Britto Ebert Falcón Guerrero artdent2000@hotmail.com
Fecha de recepción: 05 de octubre de 2017

Fecha de aceptación: 05 de noviembre de 2017 